\title{
The prognostic significance of preoperative serum cancer antigen 15-3 levels in endometrial carcinomas
}

Emre E. Tas, MD, PhD, Ayse F. Yavuz, MD, PhD.

\begin{abstract}
الأهداف : لتحديد الارتباط بين مستويات المُستضدّ المصلي للسرطان

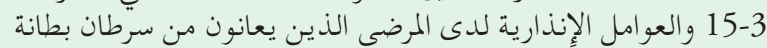

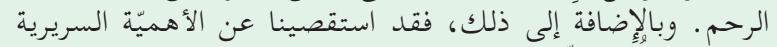

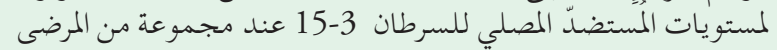

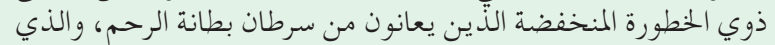

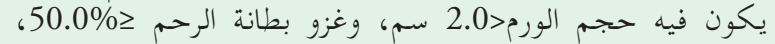

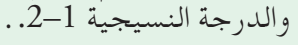

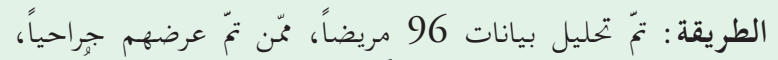

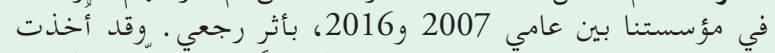

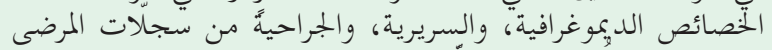

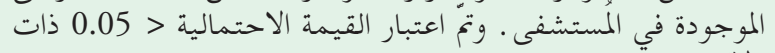
دلالة.

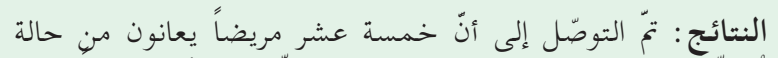

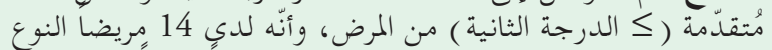

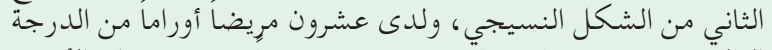

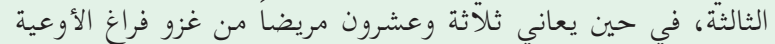

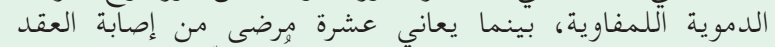

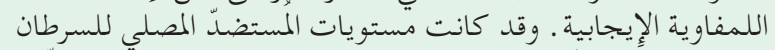

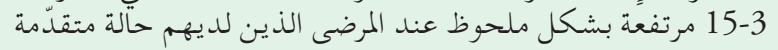

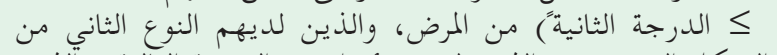

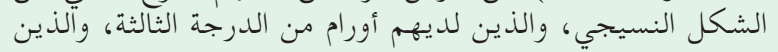

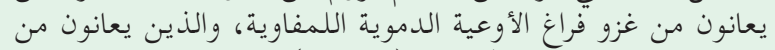

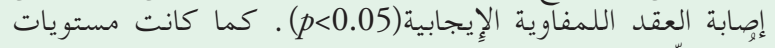

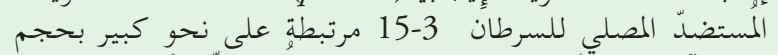

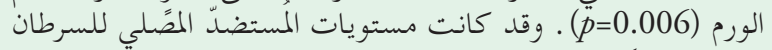

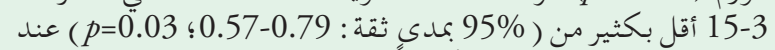

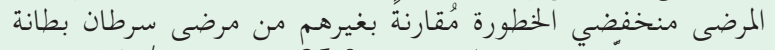

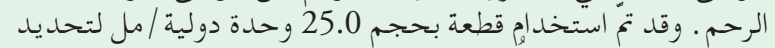

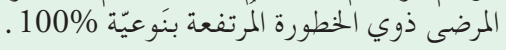

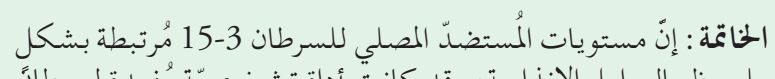

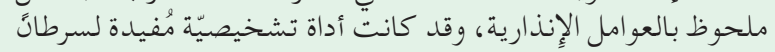
بطانة الرحم.
\end{abstract}

Objectives: To determine the associations between serum cancer antigen 15-3 levels and prognostic factors in patients with endometrial carcinomas. Additionally, we investigated the clinical utility of serum cancer antigen 15-3 levels in the selection of low-risk patients with endometrioid type, tumor size $<2 \mathrm{~cm}$, myometrial invasion $\leq 50 \%$, and histological grade 1-2.

Methods: Ninety-six patients, who were surgically staged at Ankara Yildirim Beyazit University, Ankara, Turkey, between 2007 and 2016, were retrospectively analyzed. Demographic, clinical, and surgical characteristics were retrieved from the patients' hospital records. A $p<0.05$ was considered significant.

Results: Fifteen patients had advanced ( $\geq$ Stage II) disease, 14 patients had Type 2 histology, 20 patients had Grade 3 tumors, 23 patients had lymphovascular space invasion, and 10 patients had positive lymph node involvement. Serum cancer antigen 15-3 levels were significantly higher in patients with advanced ( $\geq$ Stage II) disease, Type 2 histology, Grade 3 tumors, lymphovascular space invasion, and positive lymph node involvement $(p<0.05)$. Serum cancer antigen 15-3 levels were also significantly correlated with tumor size $(p=0.006)$. Serum cancer antigen 15-3 levels were significantly lower $(95 \%$ confidence interval: $0.57-0.79 ; p=0.03)$ in low-risk patients compared to other endometrial carcinoma patients. A cutoff of $25.0 \mathrm{IU} / \mathrm{mL}$ was used to identify high-risk patients with a specificity of $100 \%$.

Conclusion: Serum cancer antigen 15-3 levels significantly correlated with prognostic factors and were a useful diagnostic tool for endometrial carcinomas.

Saudi Med J 2017; Vol. 38 (11): 1096-1100 doi: 10.15537/smj.2017.11.20479

From the Department of Obstetrics and Gynecology, Faculty of Medicine, Ankara Yildirim Beyazit University, Ankara, Turkey.

Received 31st May 2017. Accepted 16th August 2017.

Address correspondence and reprint request to: Dr. Emre E. Tas, Department of Obstetrics and Gynecology, Ankara Yildirim Beyazit University, Ankara Ataturk Training and Research Hospital, Ankara, Turkey.E-mail:doctortas@yahoo.com

ORCID ID: http://orcid.org/0000-0001-6043-2700 
C ancer antigen 15-3 (CA15-3) is a tumor marker of several types of cancer (for example, breast, colon, and liver) that is derived from the MUC1 gene. ${ }^{1}$ Previous studies ${ }^{2-5}$ of endometrial carcinoma (EC) patients have shown that high serum CA15-3 levels are associated with a greater disease stage and a poorer prognosis. An additional study by Hebbar et $\mathrm{al}^{6}$ demonstrated that MUC1, MUC5B, and MUC8 expression was significantly increased in EC tissues compared to normal endometrial tissues. Endometrial carcinoma represents the second most frequent gynecological cancer worldwide. ${ }^{7}$ Seventy-five percent of EC patients who are diagnosed at an early stage have a relatively favorable prognosis compared to other types of gynecological cancer. However, its prevalence is increasing, especially due to obesity in developed countries. ${ }^{7,8}$ Currently, there is no reliable screening test for EC and screening is not recommended for women without certain conditions (for example, Lynch syndrome). ${ }^{9}$ However, in order to identify tumors at an early stage and to determine their prognosis, imaging methods, tumor markers, or both have been investigated. ${ }^{5,10,11}$ The role of serum CA15-3 in ECs has been studied for $>20$ years and some encouraging reports have been published. ${ }^{2-5,12}$ However, the clinical utility of serum CA15-3 has yet to be fully elucidated. The aim of this study was to determine the associations between serum CA15-3 levels and prognostic factors (age, disease stage, histological type and grade, tumor size, lymphovascular space invasion [LVSI], and lymph node involvement [LNI]) in patients with ECs. Additionally, we investigated the clinical utility of serum CA15-3 levels in the selection of low-risk patients with endometrioid type, tumor size $<2 \mathrm{~cm}$, myometrial invasion $\leq 50 \%$, and histological grade $1-2 .{ }^{13}$

Methods. The medical records of 136 EC patients who underwent staging surgery according to the recommendations of the International Federation of Gynecology and Obstetrics (FIGO) at the Ankara Atatürk Education and Research Hospital, Ankara Yildirim Beyazit University, Ankara, Turkey, between 2007 and 2016 were retrospectively analyzed. ${ }^{14}$ Forty patients whose preoperative serum CA15-3 levels were not examined were excluded. In total, 96 patients were enrolled in the study. All participants provided informed written consent. The study was approved by the Ethical

Disclosure. Authors have no conflict of interests, and the work was not supported or funded by any drug company.
Review Board Committee (Approval No=26379996/73) of Ankara Yildirim Beyazit University Faculty of Medicine, Ankara, Turkey. Research was conducted in accordance with the 1964 Declaration of Helsinki and its later amendments.

Demographic (age, gravidity, and parity), clinical (preoperative serum CA15-3 level and endometrial dual-layer thickness on transvaginal ultrasound examination), and pathological (histological tumor type and grade, extent of myometrial invasion, tumor size, FIGO surgical stage, and LVSI and LNI statuses) data were retrieved from the patients' charts and hospital records. Blood samples of patients were obtained prior to the surgery and the serum CA 15-3 values were determined by electrochemoluminescence immunoassay (Roche Elecsys Kits, Roche Diagnostic, Mannheim, Germany).

After associations between preoperative serum CA15-3 levels and prognostic factors of EC were examined (age, type 1 vs type 2 tumors, deep vs superficial invasion, early [Stage I] vs late [Stage II-IV] FIGO surgical stage, tumor size, histological grade, and LVSI and LNI statuses), we investigated the clinical utility of serum CA15-3 levels in the selection of low-risk patients with endometrioid type, tumor size $<2 \mathrm{~cm}$, myometrial invasion $\leq 50 \%$, and histological grade 1-2.

The Kolmogorov-Smirnov test was used to assess the normality of the data. Normally, distributed data were expressed as the means \pm standard deviations (ranges). Non-parametric data were expressed as the medians (interquartile ranges) (ranges). Independent samples t-tests, one-way analysis of variance tests, and post-hoc Tukey tests (when one-way analysis of variance tests were significant) were used to compare the groups. The relationships between serum CA15-3 levels and age, tumor size, and endometrial thickness were determined using the Spearman's and Pearson's correlation coefficients. Receiver operating characteristic curves were used to determine cutoff serum CA15-3 levels for selecting low-risk patients. All statistical analyses were conducted using Statistical Package for the Social Sciences for Windows, software version 21.0 (IBM Corp., Armonk, NY, USA). A $p<0.05$ was considered statistically significant and odds ratios and $95 \%$ confidence intervals were determined.

Results. The demographic, clinical, and pathological characteristics of all 96 patients with EC are summarized in Table 1. The mean serum CA15-3 levels were significantly higher in advanced ( $\geq$ Stage II) 
Table 1 - Demographic, clinical, and surgical characteristics of 96 endometrial carcinoma patients who underwent staging surgery.

\begin{tabular}{|c|c|}
\hline Characteristic & Patients $(n=96)$ \\
\hline \multicolumn{2}{|l|}{ Demographic } \\
\hline Age (years), mean \pm SD [range] & $59.1 \pm 10.0[40.0-84.0]$ \\
\hline Gravidity, median (IQR) [range] & $4(3)[0-16]$ \\
\hline Parity, median (IQR) [range] & $3(3)[0-15]$ \\
\hline \multicolumn{2}{|l|}{ Clinical mean $\pm S D$ [range] } \\
\hline Serum CA 15-3 level (IU/mL) & $21.6 \pm 16.4[4.0-153.0]$ \\
\hline $\begin{array}{l}\text { Endometrial dual-layer thickness } \\
(\mathrm{mm}), \text { median (IQR) [range] }\end{array}$ & $13.0(12.0)[1.0-62.0]$ \\
\hline \multicolumn{2}{|l|}{ Surgical } \\
\hline \multicolumn{2}{|l|}{ Histological type, $n(\%)$} \\
\hline Type 1 tumor (endometrioid) & $82(85.4)$ \\
\hline Type 2 tumor & $14(14.6)$ \\
\hline Serous & $7 \quad(7.3)$ \\
\hline Clear cell & $2(2.1)$ \\
\hline Mucinous & $2(2.1)$ \\
\hline Mixed (serous and endometrioid) & $2(2.1)$ \\
\hline Undifferentiated & $1(1.0)$ \\
\hline \multicolumn{2}{|l|}{ Histological grade, $n(\%)$} \\
\hline 1 & $57(59.4)$ \\
\hline 2 & $19(19.8)$ \\
\hline 3 & $20(20.8)$ \\
\hline \multicolumn{2}{|l|}{$L V S I, n(\%)$} \\
\hline Positive & $23(24.0)$ \\
\hline Negative & $73(76.0)$ \\
\hline \multicolumn{2}{|l|}{$F I G O$ stage, $n(\%)$} \\
\hline IA & $61(63.6)$ \\
\hline IB & $20(20.8)$ \\
\hline II & $4 \quad(4.2)$ \\
\hline IIIB & $1(1.0)$ \\
\hline IIIC1 & $3(3.1)$ \\
\hline IIIC2 & $3(3.1)$ \\
\hline IVA & $2(2.1)$ \\
\hline IVB & $2(2.1)$ \\
\hline Tumor size (cm), mean $\pm S D$ [range] & $3.5 \pm 2.0[0.1-11.0]$ \\
\hline \multicolumn{2}{|l|}{ Lymph node involvement, $n(\%)$} \\
\hline Yes & $10(10.4)$ \\
\hline No & $86(89.6)$ \\
\hline
\end{tabular}

tumors compared to early (Stage I) tumors $(34.2 \pm 35.3$ [7.0-153.0] $\mathrm{IU} / \mathrm{mL}$ vs $19.3 \pm 9.8$ [4.0-44.0] $\mathrm{IU} / \mathrm{mL}$; $p=0.001)$. Moreover, there was a weak linear correlation between serum CA15-3 levels and tumor size (Spearman's correlation coefficient, $p=0.006$ ) (Figure 1 ). In contrast, there were no associations between serum CA15-3 levels and age (Spearman's correlation coefficient, $p=0.78$ ) or endometrial dual-layer thickness (Pearson's correlation coefficient, $p=0.60$ ).

The mean serum CA15-3 levels were significantly higher in histological Type 2 tumors compared to histological Type 1 tumors $(31.8 \pm 37.2$

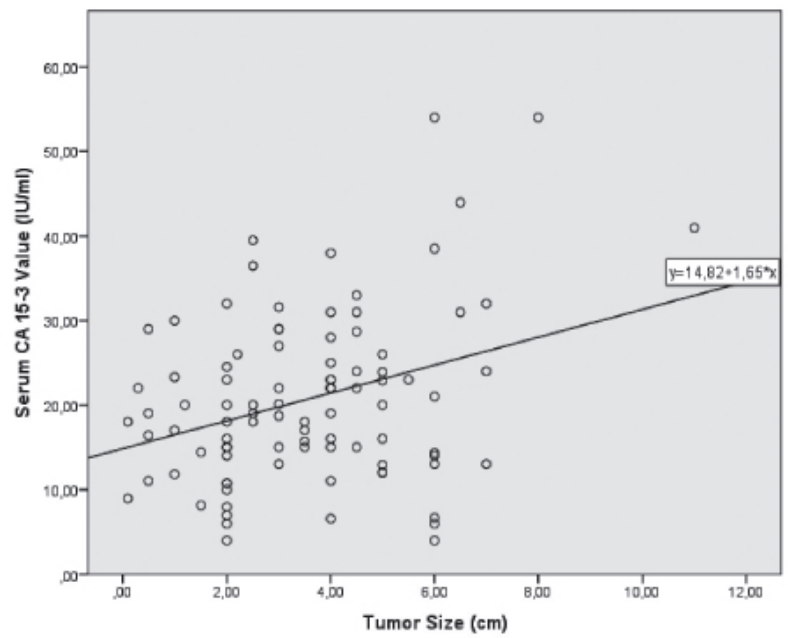

Figure 1 - Spearman's correlation between serum cancer antigen (CA15-3) levels and tumor size ( $p=0.006)$. Serum CA15-3 levels of $>55.0 \mathrm{IU} / \mathrm{mL}$ were fixed to $55.0 \mathrm{IU} / \mathrm{mL}$.

[6.7-153.0] IU/mL vs $19.9 \pm 8.6[4.0-41.0] \mathrm{IU} / \mathrm{mL}$; $p=0.01)$. Thereafter, mean serum CA15-3 levels differed significantly according to histological grade $(p=0.007)$. Post-hoc Tukey analyses revealed that the mean serum CA15-3 levels were significantly higher in Grade 3 tumors $(31.7 \pm 30.7[4.0-153.0] \mathrm{IU} / \mathrm{mL})$ compared to Grade $1(17.9 \pm 7.8$ [8.0-38.0] IU/mL; $p=0.009)$ or Grade 2 tumors (19.4 \pm 8.5 [4.0-41.0] IU/mL; $p=0.02)$. In contrast, no significant differences were observed between Grade 1 and Grade 2 tumors ( $p=0.90$ ).

The mean serum CA15-3 levels were significantly higher in patients with LVSI or LNI compared to patients without LVSI or LNI $(29.0 \pm 29.4$ [4.0-153.0] IU/mL vs $19.3 \pm 8.3[4.0-41.0] \mathrm{IU} / \mathrm{mL}[p=0.01]$ and $36.5 \pm 42.9$ $[10.7-153.0] \mathrm{IU} / \mathrm{mL}$ vs $19.9 \pm 8.8[4.0-44.0] \mathrm{IU} / \mathrm{mL}$ $[p=0.002])$.

Finally, the mean serum CA15-3 levels were lower in low-risk patients compared to other patients with ECs (16.2 \pm 7.1 [4.0-32.0] IU/mL vs $24.4 \pm 18.2$ [4.0-153.0] $\mathrm{IU} / \mathrm{mL})$. This difference was statistically significant (95\% confidence interval: 0.57-0.79; $p=0.03$ ). Receiver operating characteristic curve analysis revealed that the most suitable cutoff serum CA15-3 level for discriminating between low-risk patients and other patients with ECs was $18.5 \mathrm{IU} / \mathrm{mL}$. The sensitivity was $64 \%$ and the specificity was $67 \%$ (Figure 2). When the groups (low-risk patients vs other patients with ECs) were assessed in the receiver operating characteristic curve analysis in terms of the $25.0 \mathrm{IU} / \mathrm{mL}$ cutoff value, the sensitivity was $33.3 \%$ and the specificity was $100 \%$. 


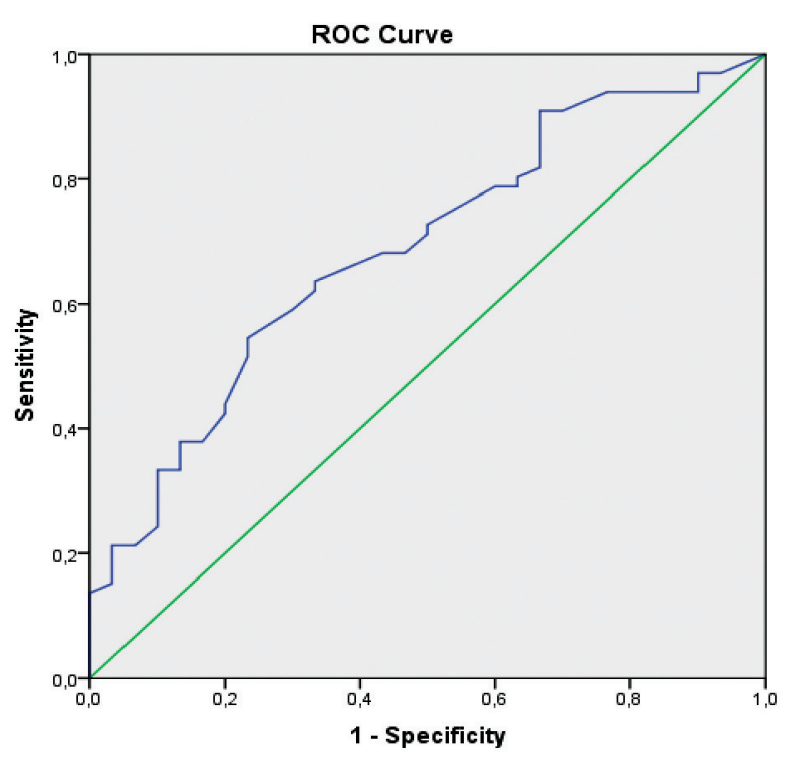

Figure 2 - Receiver operating characteristic curve of serum cancer antigen 15-3 levels for discriminating low-risk patients (endometrioid type, tumor size $<2 \mathrm{~cm}$, myometrial invasion $\leq 50 \%$, and histological grade 1-2) from other patients with endometrial cancer (area under curve: 0.68; standard error: 0.06).

Discussion. The usefulness of different serum tumor markers has been investigated in EC patients for $>20$ years. It has been suggested that the application of serum tumor markers with or without the use of different techniques (namely, imaging or pathological procedures) may reflect tumor stage and prognosis in a number of studies. ${ }^{2-5,10,11}$ However, inconsistent results have been published and there is no consensus on whether serum tumor markers are of diagnostic or prognostic importance in EC. Studies examining serum CA15-3 levels in EC patients - $-5,12,15$ were also found to be inconsistent.

In several preliminary reports, Soper et $\mathrm{al}^{2}$ and Scambia et $\mathrm{al}^{3}$ had demonstrated that advanced tumor stage and extra-uterine disease risks were increased with elevated serum CA15-3 levels. Moreover, high serum CA15-3 levels were associated with a number of poor prognostic factors (for example, histological grade) and a shorter survival. ${ }^{3,5,12,16,17}$ However, no consensus has been reached on whether these differences are significant or not.

In contrast to previous reports, we determined preoperative serum CA15-3 levels to be significantly higher in advanced ( $\geq$ Stage II) EC patients compared to early (Stage I) EC patients $(p<0.05) .{ }^{4}$ However, due to insufficient patient numbers, we were unable to determine whether serum CA15-3 levels differed between advanced FIGO stages. It has been well established that certain factors (for example, older age, advanced tumor grade, type 2 histology, tumor size, and positive LVSI and LNI statuses) are independent prognostic factors for EC. ${ }^{18}$ However, the relationships between serum CA15-3 levels and these prognostic factors have yet to be fully elucidated. In the present study, we failed to demonstrate an association between serum CA15-3 levels and age. However, we did find that serum CA15-3 levels differed significantly according to tumor size, histological type (Type $2>$ Type 1), histological grade (Grade $3>$ Grade 1-2), and positive LVSI and LNI statuses. In previous studies, Scambia et $\mathrm{al}^{3}{ }^{3} \mathrm{Li}$ et $\mathrm{al}^{5}$ and Yildiz et $\mathrm{al}^{19}$ reported significant associations between serum CA15-3 levels and FIGO stage, histological grade, and a positive LNI status. However, we demonstrate significant associations between serum CA15-3 levels and tumor size, histological type, and a positive LVSI status.

Low-risk patients (endometrioid type, tumor size $<2 \mathrm{~cm}$, myometrial invasion $\leq 50 \%$, and histological grade 1-2) have a favorable prognosis and it is still debated whether comprehensive surgery is necessary in these patients. ${ }^{20,21}$ However, the successfulness of investigations that have been performed using different techniques (for example, preoperative imaging studies with or without serum tumor markers and in traoperative frozen section analysis) to discriminate between lowand high-risk patients has varied considerably. ${ }^{10,11,22}$ Moreover, serum CA15-3 levels have not been evaluated in terms of selecting these patients. In the present study, we demonstrate that serum CA15-3 levels differ significantly between low- and high-risk patients and that $18.5 \mathrm{IU} / \mathrm{mL}$ is the most suitable cutoff value for patient selection (sensitivity, 64\%; specificity, 67\%). Furthermore, a cutoff value of $25.0 \mathrm{IU} / \mathrm{mL}$ was able to identify patients in need of comprehensive staging surgery with a specificity of $100 \%$.

In conclusion, although this study has several limitations (for example, its retrospective single-center design and relatively small sample size), we propose that serum CA15-3 levels are a useful diagnostic tumor marker for EC. Comprehensive studies on different histological subtypes are necessary to determine the utility of this tumor marker for patients with ECs.

\section{References}

1. Xu F, Liu F, Zhao H, An G, Feng G. Prognostic significance of mucin antigen MUC1 in various human epithelial cancers: A meta-analysis. Medicine (Baltimore) 2015; 94: e2286. 
2. Soper JT, Berchuck A, Olt GJ, Soisson AP, Clarke-Pearson DL, Bast RC Jr. Preoperative evaluation of serum CA 125, TAG 72, and CA $15-3$ in patients with endometrial carcinoma. Am J Obstet Gynecol 1990; 163: 1204-1209.

3. Scambia G, Gadducci A, Panici PB, Foti E, Ferdeghini M, Ferrandina G, et al. Combined use of CA 125 and CA 15-3 in patients with endometrial carcinoma. Gynecol Oncol 1994; 54 : 292-297.

4. Baser E, Gungor T, Togrul C, Turkoglu O, Celen S. Preoperative prediction of poor prognostic parameters and adjuvant treatment in women with pure endometrioid type endometrial cancer: what is the significance of tumor markers? Eur J Gynaecol Oncol 2014; 35: 513-518.

5. Li J, Lin J, Luo Y, Kuang M, Liu Y. Multivariate analysis of prognostic biomarkers in surgically treated endometrial cancer. PloS One 2015; 10: e0130640.

6. Hebbar V, Damera G, Sachdev GP. Differential expression of MUC genes in endometrial and cervical tissues and tumors. BMC Cancer 2005; 5: 124.

7. Creasman WT, Odicino F, Maisonneuve P, Quinn MA, Beller U, Benedet JL, et al. Carcinoma of the corpus uteri. FIGO 26th Annual Report on the Results of Treatment in Gynecological Cancer. Int J Gynaecol Obstet 2006; 95 Suppl 1: 105-143.

8. Torre LA, Bray F, Siegel RL, Ferlay J, Lortet-Tieulent J, Jemal A. Global cancer statistics, 2012. CA Cancer J Clin 2015; 65: 87-108.

9. Lu KH, Daniels M. Endometrial and ovarian cancer in women with Lynch syndrome: update in screening and prevention. Fam Cancer 2013; 12: 273-277.

10. Andreano A, Rechichi G, Rebora P, Sironi S, Valsecchi MG, Galimberti S. MR diffusion imaging for preoperative staging of myometrial invasion in patients with endometrial cancer: a systematic review and meta-analysis. Eur Radiol 2014; 24: 1327-1338.

11. Kang S, Nam JH, Bae DS, Kim JW, Kim MH, Chen X, et al. Preoperative assessment of lymph node metastasis in endometrial cancer: A Korean Gynecologic Oncology Group study. Cancer 2017; 123: 263-272.

12. Lo SS, Cheng DK, Ng TY, Wong LC, Ngan HY. Prognostic significance of tumour markers in endometrial cancer. Tumour Biol 1997; 18: 241-249.
13. Milam MR, Java J, Walker JL, Metzinger DS, Parker LP, Coleman RL, et al. Nodal metastasis risk in endometrioid endometrial cancer. Obstet Gynecol 2012; 119: 286-292.

14. Amant F, Mirza MR, Koskas M, Creutzberg CL. Cancer of the corpus uteri. Int J Gynaecol Obstet 2015; 131: 96-104.

15. Żyła MM, Wilczyński JR, Kostrzewa M, Księżakowska-Łakoma K, Nowak M, Stachowiak G, et al. The significance of markers in the diagnosis of endometrial cancer. Prz Menopauzalny 2016; 15: 176-185.

16. Cherchi PL, Dessole S, Ruiu GA, Ambrosini G, Farina M, Capobianco, et al. The value of serum CA 125 and association CA 125/CA 19-9 in endometrial carcinoma. Eur J Gynaecol Oncol 1999; 20: 315-317.

17. Mylonas I, Mayr D, Walzel H, Shabani N, Dian D, Kuhn C, et al. Mucin 1, Thomsen-Friedenreich expression and galectin-1 binding in endometrioid adenocarcinoma: an immunohistochemical analysis. Anticancer Res 2007; 27: 1975-1980.

18. Hacker NF, Friedlander MY. Uterine Cancer. In: Berek JS, Hacker NF, editors. Berek \& Hacker's Gynecologic Oncology, 6th Edition. Philadelphia (PA): Wolters Kluwer; 2015. p. 390-442.

19. Yildiz A, Ozcan A, Yetimalar MH, Kasap B, Yigit S, Kilic Sakarya D, et al. The predictability of lymph node metastasis in patients with endometrial cancer. Turkiye Klinikleri J Med Sci 2013; 33: 1028-1036.

20. Hidaka T, Nakashima A, Shima T, Hasegawa T, Saito S. Systemic lymphadenectomy cannot be recommended for low-risk corpus cancer. Obstet Gynecol Int 2010; 2010: 490219.

21. Kokcu A, Kurtoglu E, Celik H, Kefeli M, Tosun M, Onal M. Is surgical staging necessary for patients with low-risk endometrial cancer? a retrospective clinical analysis. Asian Pac J Cancer Prev 2015; 16: 5331-5335.

22. Alcazar JL, Dominguez-Piriz J, Juez L, Caparros M, Jurado M. Intraoperative Gross examination and intraoperative frozen section in patients with endometrial cancer for detecting deep myometrial invasion: a systematic review and meta-analysis. Int J Gynecol Cancer 2016; 26: 407-415. 